\title{
Active IR-Thermal Imaging in Medicine
}

\author{
Mariusz Kaczmarek $^{1}$ • Antoni Nowakowski ${ }^{1}$
}

Received: 14 August 2015 / Accepted: 9 January 2016 / Published online: 29 January 2016

(C) The Author(s) 2016. This article is published with open access at Springerlink.com

\begin{abstract}
In this paper we summarize results of several research projects devoted to development of new diagnostic methods and procedures based on quantitative infrared thermography in medical applications. First, basics of active dynamic thermography are presented. Described are both, instrumentation and software comprising measurement procedures including collection of series of IR-images after external excitation, data treatment with displacement corrections, reduction of noise and artifacts and finally presentation of parametric diagnostic images. The thermal tomography method is also shortly discussed. As the most important for medical diagnostics parametric images of thermal time constants are discussed. For illustration of the value of presented methods chosen research and clinical applications performed in several clinics of Gdansk Medical University are presented. In vivo experiments on animals, mainly domestic pig, as well as clinical diagnostic procedures are discussed in skin burn and cardiosurgery applications. Specific requirements of IR-thermal investigation in medical applications are discussed in comparison to IR-thermal imaging in technical non-destructive evaluation. It should be underlined that medical applications of IR-diagnostics seem to be the most difficult among other applications as heat transfers in living tissues are far more complicated comparing to technical
\end{abstract}

This article is part of the Topical Collection on Thermography.

Mariusz Kaczmarek

mariusz.kaczmarek@eti.pg.gda.pl

Antoni Nowakowski

antowak@biomed.eti.pg.gda.pl

1 Department of Biomedical Engineering, Faculty of

Electronics Telecommunication and Informatics,

Gdansk University of Technology, Narutowicza 11/12,

80-233 Gdansk, Poland evaluation where nondestructive testing methods based on thermal IR-imaging are already well developed.

Keywords Dynamic thermography - Diagnostics \& evaluation $\cdot$ Thermal descriptors · Medical applications

\section{Introduction}

State-of-the-art in quantitative infrared (IR) thermography (QIRT) in medical applications in comparison to equivalent non-destructive evaluation (NDE) methods developed for technical testing is here presented. Very broad overview of non-destructive testing NDT and NDE methods mainly in industrial engineering and other technical applications is given by Maldague in the monograph [1] and recently by Vavilov in the review [2].

Diagnostic role of temperature in medicine is known for ages - already Hippocrates (ca 480 B.C.) was able to recognize hot and cold spots on patient's body by drying mud slurry spread over the patient. The state of the art of thermal imaging in medicine is broadly discussed in the monograph [3], where Ring [4] discussed the historical background of thermal diagnostics and Amalu and others made the review of IR imaging of the breast [5]. Already in sixties of XX century, adapting military cameras doctors Bowling Barnes [6] and Gershen-Cohen [7] started the use of static IR-thermography in medicine, especially showing possibility of breast cancer imaging but also other applications. Further developments were possible due to availability of fast, based on two rotating prisms and a single InSb cooled detector, real time operation AGA cameras (name of the Swedish company, later called AGEMA, at present FLIR), what allow in early seventies XX century in Sweden and in the USA to start programs for mass screening of breast cancer patients. Unfortunately early enthusiastic reception of thermography and its mas- 
sive use in breast cancer diagnostics was rapidly stopped after negative conclusions of the Breast Cancer Detection and Demonstration Project [5], mainly due to many errors caused by unqualified personnel. In fact though investigation using IR-cameras is absolutely non-invasive and aseptic the thermal signal is not specific and interpretation of IR images requires strictly defined measurement conditions what was not fully understood at this time. Since this moment one may observe real development of IR-imaging technology, introduction of QIRT methods and computer simulation allowing better understanding of thermal processes.

Today high quality cameras are broadly accessible in reasonable prices what makes QIRT an attractive diagnostic option in many fields of applications. Unfortunately early critical opinion of thermography as a diagnostic tool in breast cancer is still present among cancer specialists, though this opinion should be changed. Recent works show, that none of existing diagnostic methods, even the reference X-ray mammography, is perfect and only matching of different modalities allows increasing the proper diagnosis level to $98 \%$ [8]. This is due to limited value of each of diagnostic modalities. X-ray mammography, ultrasound and NMR (nuclear magnetic resonance) imaging show structural changes characteristic for well developed cancerous processes while IR-imaging provides a reflection on metabolic activity what allows for early detection of cancer symptoms, though temperature is not a specific Figure of Merit. Functional tumour induced angiogenesis is leading to development of vascularization in the region of tumour formation even several years before any other tissue parameters are changed. This is proved by some other investigations, as e.g. electrical bioimpedance, too. Fortunately, IR-imaging that is absolutely non-invasive and non-destructive modality allowing frequent repetition of patient testing, was recently approved by FDA as an auxiliary modality for mammographic screening.

During last 25 years till now in parallel to classical functional static thermography ST several non-destructive methods of active thermography (AT), as lock-in, pulsed phase thermography (PPT), thermographic signal reconstruction (TSR) and active dynamic thermography (ADT) are in the phase of rapid development. All those methods significantly differ comparing to ST allowing for determination of thermal properties of a tested region, giving structural data. It should be underlined that classical IR-thermography is a functional imaging-distribution of temperature reflects metabolic processes-while AT methods are showing distribution of tissue thermal properties, what gives structural information. Matching both modalities strongly improves value of thermal diagnostics. What is more, using the same IR-camera two different modalities are available providing functional as well as structural data, what is strongly implementing diagnostic information.
Rapid development of AT applications is possible due to increased availability of the IR-imaging technology caused by significant decrease of equipment prices and increased availability of mature technology based on uncooled FPA (focal plane array) IR-detectors as well as by implementation of advanced digital image analysis methods. The new developments in this field include also approaches to thermal tomography (TT) and to visualisation using stereovision thermal systems. Here we show advances of active thermography in medicine, and especially we discuss specific conditions while dealing with living tissue.

It should be underlined that medical applications of QIRT comparing to technical NDE are much more difficult for interpretation as heat transfers in living tissues are far more complicated comparing to technical applications. First, the human body is a very complicated structure, alive and geometrically flexible; second, thermal boundary conditions are usually not fully known and what is more, temperature distribution inside a tested structure usually is not uniform. Additionally natural biofeedback dynamic reactions of living organisms and tissues may be strong and should be avoided to get clear interpretation of measurement results.

One important feature of ADT in medicine should be underlined-analysis of a given problem requires high skills in modelling of a tested structure as all processes of diagnostics are funded on objective model based evaluation of measurement data. This is in contradiction to expectations of medical staff to have diagnostic tools easy in operation and fully automatic. There are also other problems, which have to be taken into account to perform reliable analysis of measurement data, as elimination of displacement errors, choice of proper measurement conditions etc. Matching all such expectation is not easy and requires advanced software development. The following text contains discussion of problems present in ADT IR-thermal analysis of the living species.

In this paper experiences of the Department of Biomedical Engineering Gdansk University of Technology in cooperation with several clinics of Gdansk Medical University in frontal edges of ADT in medical applications are discussed. Basic problems related to practical applications of QIRT in medical diagnostics - in burn diagnostics, in quality evaluation of cardiosurgery procedures, evaluation of post-surgery wound healing and others-are presented. First, we discuss technical problems related to instrumentation as well as to digital signal processing and data analysis, second, several practical applications are discussed.

\section{Instrumentation and Measurement Procedures}

As it was already underlined both, classical functional thermography ST, using a high quality IR-thermal camera, and active dynamic thermography ADT procedures are applied together for medical diagnostics. Usually the results of mul- 


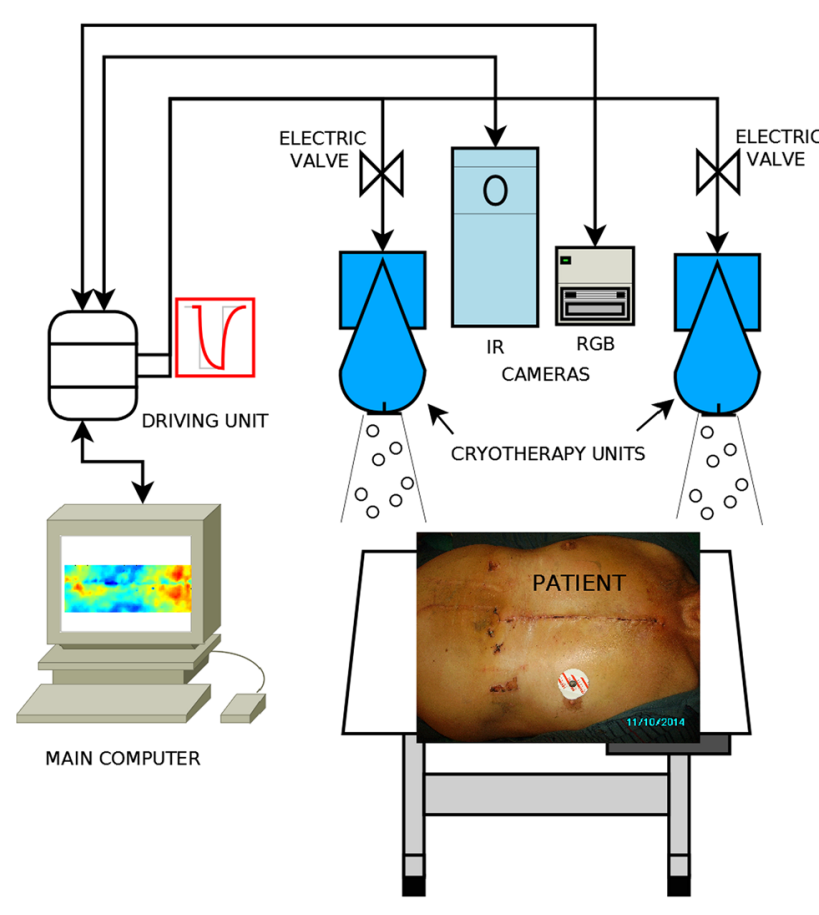

Fig. 1 Typical measurement set for ADT experiments with cooling excitation

timodality investigations and analysis are matched, allowing complex diagnostic approach and improved classification of affected regions, as e.g. burns requiring surgical treatment.

A versatile measurement set combining instrumentation for ST and ADT methods is shown in Fig. 1. In ADT changes in tested object surface temperature are caused by external heating or cooling and are dependent on the internal structure and thermal properties of a tested object. In most of medical applications the cooling procedure is applied as it is shown in Fig. 2 by switching-on the cooling devices. We used in our experiments different cryotherapy units. One of the best is the unit using expanded liquid $\mathrm{CO}_{2}$ gas mixed with ambient air, allowing to set a wide range of the temperature of a cooling stream (from ca. $0^{\circ} \mathrm{C}$ up to the ambient temperature). It's main advantage is fully aseptic mode of operation, formally accepted for clinical use. The other solutions used to be an industrial air conditioning unit as well as air cryotherapy clinical system. In both cooling is due to the stream of the ambient air (from $5^{\circ} \mathrm{C}$ up to the ambient temperature), unfortunately an air filter must be applied and often exchanged to keep aseptic conditions of cooling. Generally, for cooling using a stream of gas the heat exchange occurs mainly due to forced convection.

In our experiments two identical cryotherapy units are used to allow relatively uniform cooling in the region of interest ROI. Typically cooling lasts 30-60 s, till surface temperature in the ROI is reaching the level of the room temperature, when the cryo-units are switched-off, Fig. 2a. From this moment registration of the ROI temperature is

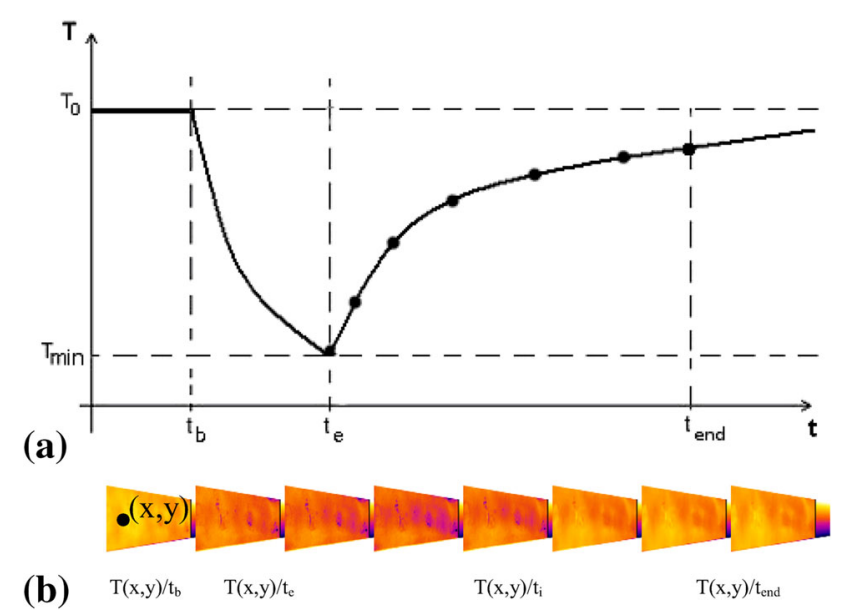

Fig. 2 ADT measurement procedure-registration of temperature transients at any pixel $(x, y)$ in time; a external cooling; $t_{b / e}$-moments the source is switched on (beginning) and off (end of excitation), $t_{\text {end }}$ time of termination of the recording; $\mathbf{b}$ recorded temperature distribution

performed, typically during the period equal three times of the cooling time, Fig. 2b. IR camera allows the surface temperature to be recorded at a required speed, rated in frames/second. Thermal transients are recorded at the surface of a tested region, usually after rectangular external excitation, to avoid interaction with excitation media, so registration is performed during the phase of relaxation - natural return to the steady state conditions-the points indicated in Fig. 2b. Generally, ADT allows determination of thermal tissue properties, specific e.g. for different depths of burn wounds; the state of healing of surgical wounds, and many other clinical cases.

As it is shown in Figs. 1 and 3 visual cameras (indicated as RGB) may be applied for optical inspection and stereovision experiments to allow full documentation of the diagnostic procedure. Typically at the beginning static thermal and visible images are registered giving reference data.

Full multimodality, advanced experiment may be performed in the configuration shown in Fig. 3-a block diagram of a set, allowing 3-D experiments. In full configuration two IR and two RGB cameras are applied. Additionally an electrocardiograph-ECG monitor with the QRS-detector may be applied to allow registration of images synchronized with the heart action in cardiosurgery experiments. Laser pointers are used for proper placement of cooling streams to allow relatively uniform cooling of a patient as it is shown in Fig. 4.

Diagnostic information in both modalities ST and ADT is of different character. Classical thermography shows regions of increased or decreased temperature, usually caused by change of vascularisation affected due to different clinical reasons as cancer, wound, other causes of increased or decreased metabolism, necrosis, ischemia etc. ADT allows 
Fig. 3 Double cameras (IR and RGB) synchronised with an excitation source allow to perform 3-D study, other elements are dependent on application, e.g. in cardiosurgery a unit for synchronisation of recordings with the heart rate is necessary-here the ECG monitor

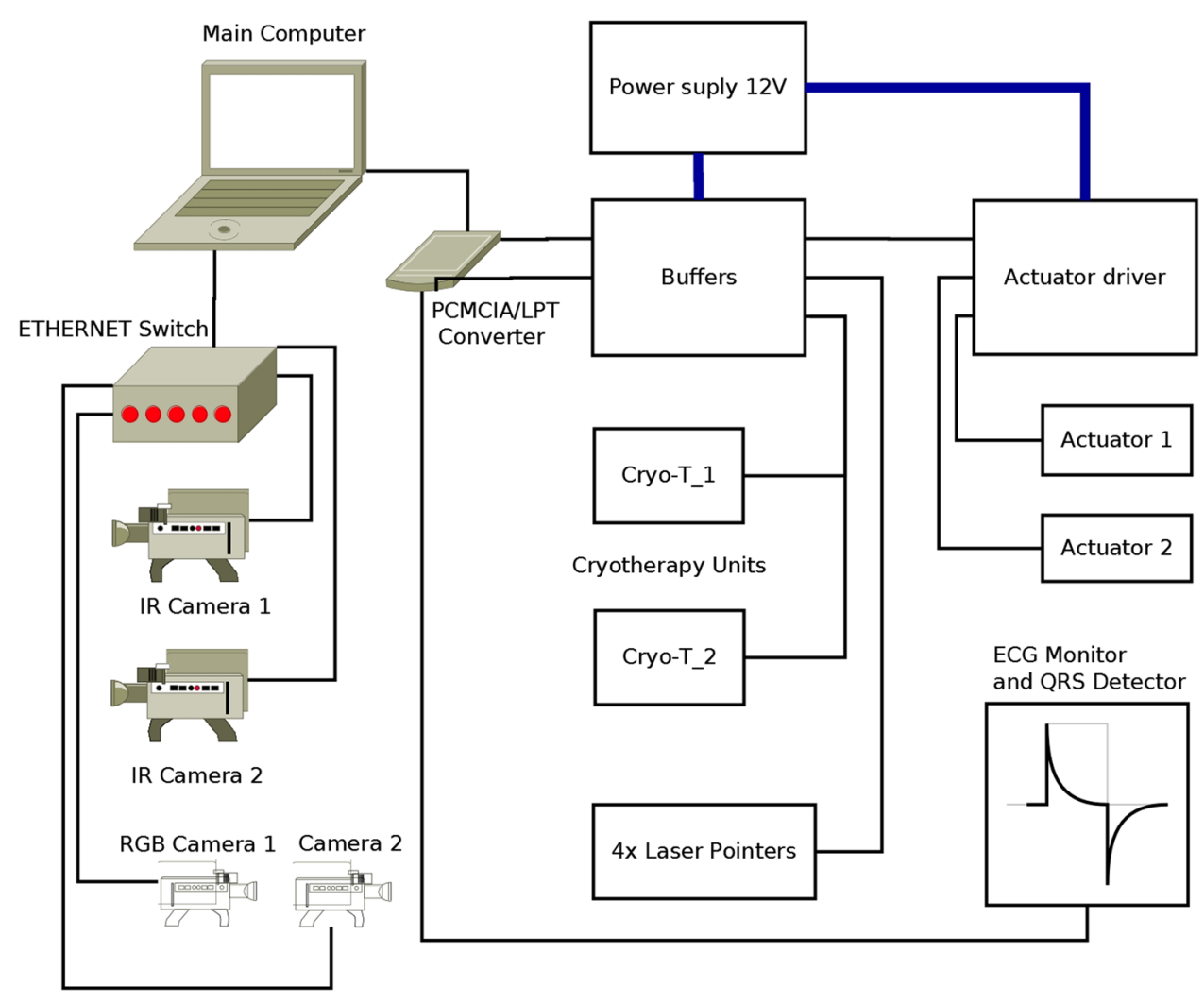

determination of thermal properties of a tested region by analysing the transient processes which are correlated with physical (thermal) tissue properties such as thermal conductivity and capacity, tissue density, water content, vascularization and heat transfer by flowing blood etc.

The minimal requirements for IR-thermal camera are: resolution below $0.1^{\circ} \mathrm{C}$; speed of operation not less than 30 frames per second and the lens field of view FOV adequate for chosen application. Choice of a short wavelength SW or long wavelength LW camera is of secondary importance, as modern thermal cameras in both ranges of radiation are of similar thermal resolution and speed of operation. The construction of a versatile ADT system applied in discussed measurements is shown in Fig. 4.

Important is control of the shape and time of the external thermal excitation. For medical applications, to avoid biofeedback, a rectangular pulse lasting no more than one minute seems to be the best. To our practical experience in such conditions the influence of the thermoregulatory mechanism is minimal. So, depending on application the energy and time of such excitation should be controlled. While heating, the surface temperature should never exceed $42^{\circ} \mathrm{C}$ to avoid tissue damage. While cooling, the best is the condition of reaching the level of the room temperature, allowing controlled heat flows - in this condition only internal flows may exist as there is no temperature gradient for heat exchange with the environment.

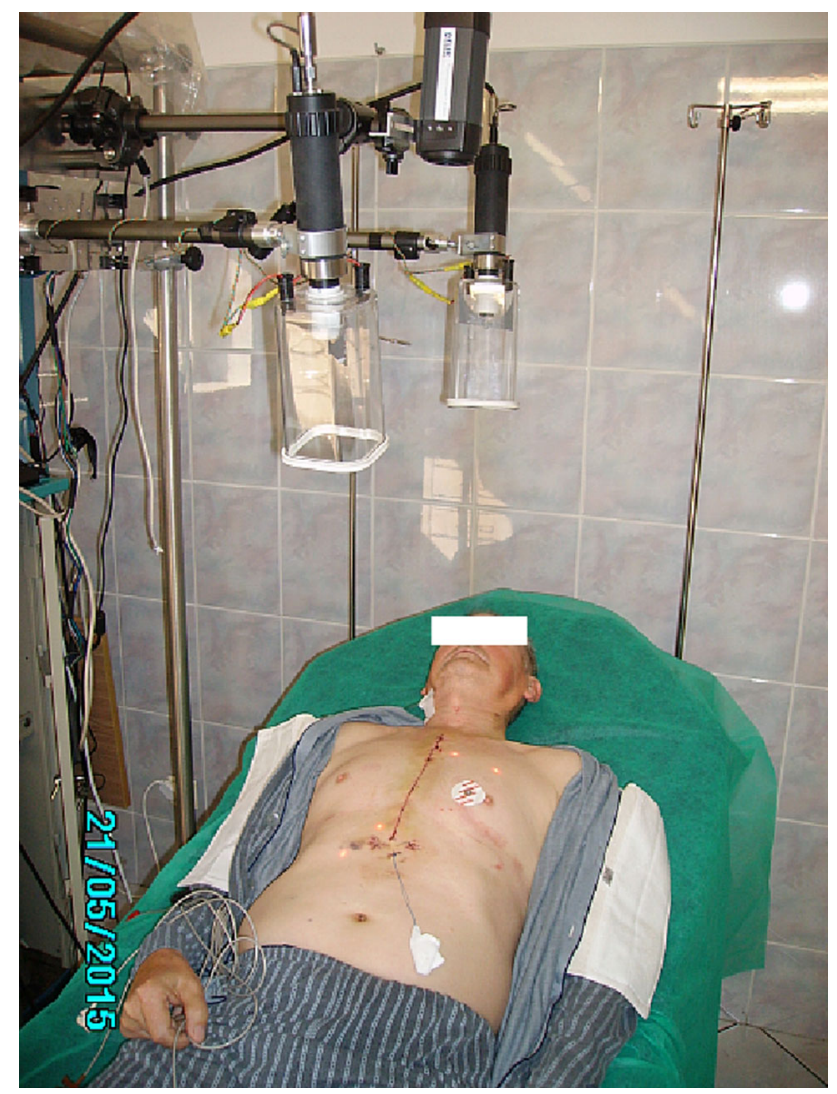

Fig. 4 The experimental ADT set-up in clinical environment, visible are cooling heads and IR-camera (LW FPA, uncooled) 
Usually thermal processes at the ROI surface may be approximated by exponential models. Therefore registration of transient thermal distribution at the surface of a tested object allows calculation of such Figures of Merit as thermal time constants and presentation each in the form of parametric images. Application of additional numerical procedures as TSR or Thermal Tomography allow further improvement of diagnostics allowing tested region geometry reconstruction and giving high quality recognition of the affected region, e.g. determination of the depth of a burn or objective description of healing processes.

\section{Software Problems}

There are several levels of the software necessary to perform data acquisition and analysis leading to diagnostic conclusions. First, a specific software for execution of all necessary actions of the system, as registration of static images, starting measurements, synchronisation of excitation sources and recording cameras, synchronisation of different cameras if several are applied - visible and IR, especially in stereovision experiments etc. It combines all elements of communication interfaces, including user-friendly manipulation of the system (graphic user interface GUI). Usually a C++ or C\# software specific for the technology is applied. We use for these purposes our own software applications (C\# and MS Visual Studio). In ADT capturing a sequence of images after thermal excitation is followed by a fitting procedure with pre-processing (including motion compensation and noise filtering) and post processing (extraction of parametric images, ROI determination etc.) for proper visualization of the results. This phase is done in MatLab environment for prototyping and then written in $\mathrm{C} \#$ for final application. This is illustrated on the Fig. 5.

Two main factors are influencing measurements based on series of images: first, unintentional displacements of a patient; second, changes of the thermal content in following images. This leads to the question how to analyze thermal transients and to apply procedures of automatic understanding of image content, if each detector of FPA sees in following moments not the same region and additionally temperature is also changed? Therefore the second level software (see Fig. 5b) is devoted to solve practical problems, as to match images from different modalities or to compare images taken during following in time examinations. To eliminate natural movements of a living object helpful is synchronisation of images, e.g. with breathing, but this problem is especially important in the case of cardiosurgery investigations when synchronization with the heart rate is absolutely necessary. In fact dynamic action of the heart in time combine not only changes of the position but also the angle of observation, volume and temperature at the surface of the heart, limiting possibility of using ADT for proper diagnostics of the state of the heart muscle [9].

The first action must be matching of following images to see the same area of a tested object/region, to allow further thermal analysis of series of thermal images. This task is not always easy, especially when the tested object is moving its position and shape in time. Usually thermal images contain less geometry information comparing to visible images; therefore often matching of both images IR and visible may help in automatic understanding of thermal content.

Generally matching of images is not an easy task. One has to find as much as possible of controlled points well recognised in both images. For further correction of images the morphing transforms or other method, as e.g. FFDfree form deformation should be applied [10]. Image and sequence preprocessing consists of image matching for ROI translations elimination, detection of the excitation range and time transient segmentation for determining exact parameters of the temperature curve. For thermal images we are using mostly the fast fourier transform FFT spectra based on direct phase substitution (DPS) algorithm that consist of smooth temperature gradients rather than distinctive features like edges and corners [11]. Matching of images in (a)

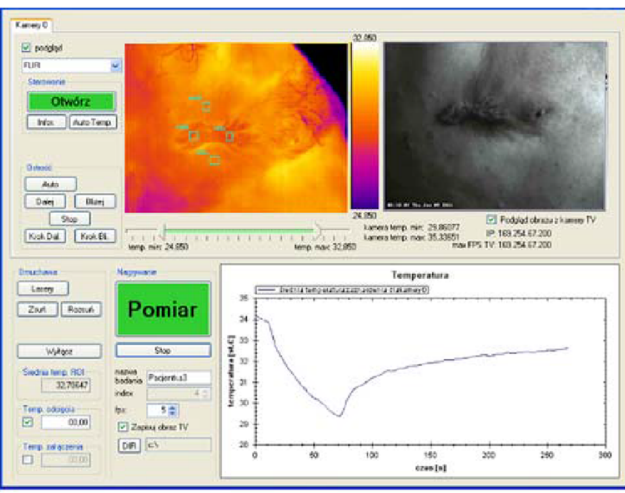

(b)

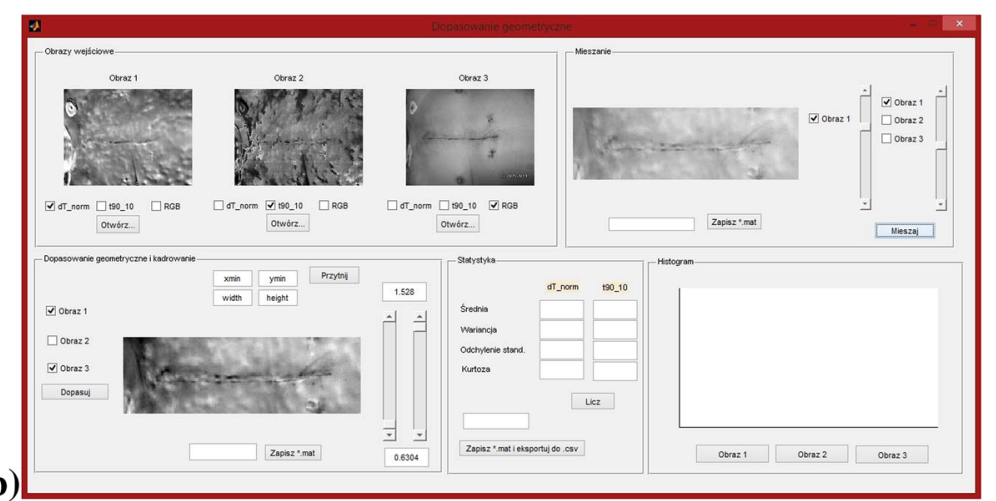

Fig. 5 Application GUI for the ADT procedures, a controll software for the set shown in Fig. 3-steering of devices and sequence of images capturing; b second level software for post-processing, as fitting images, ROI determination, displacement corrections etc. 
Fig. 6 The block diagram of tomography

procedure-comparing of experiment with simulation-in TT an equivalent thermal model is modified in iterative procedure

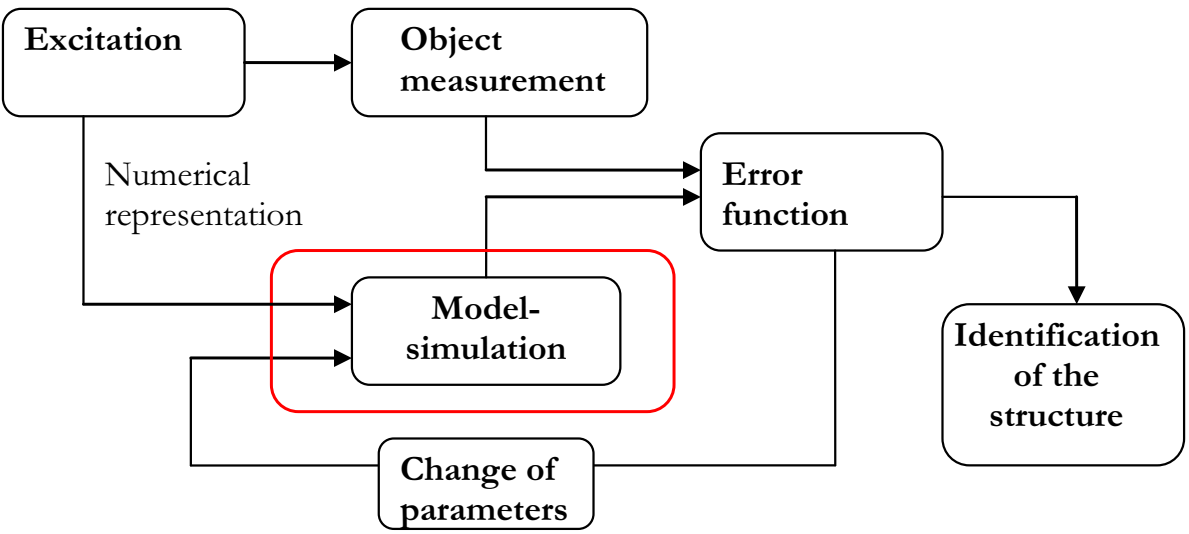

the way that the tested object stays in the unchanged position and shape strongly improves quality of analysis of thermal image series and decreases the errors in further diagnostics.

The main condition for effective application of ADT in diagnostics is reliable recognition of a tested organ and automatic transformation of series of images collected in time to the initial dimensions of a region of interest. In this case the main problem is interpretation of temperature distribution and thermal transients at the surface of a tested object after external excitation in ADT experiments. Theoretically full answer (reconstruction and determination of 3-D geometry) may be given by solving the thermal tomography problem TT, according to classical tomography procedure shown in Fig. 6. Here basic is the role of thermal models of the tested object. In fact TT, that usually is an iterative procedure, aims to reconstruct thermal properties of the tested object. It is an extremely difficult problem, as mathematically it is ill posed and usually boundary conditions are rather not known. Please do remember, that in biological living objects not only the structure is extremely complicated, with such elements as skin, arteries, veins, fat, bones etc. but additional complication relates to metabolic processes that are responsible for heat generation and non-uniform temperature distribution along the tissue. Typically temperature at the skin surface is of a few degrees lower than internal body temperature.

Very important step in TT procedure is solution of the forward problem-the model-simulation part (heat transfer simulations). This step is executable many times, so it has main influence on time and accuracy of the whole process of thermal parameters determination and the tested structure reconstruction.

The obvious solution is application of the 3-D model of a tested object to be used for analysis of heat flows. Typically the heat equation and a FEM model are applied using different software tools, as e.g. COMSOL and MatLab environment. One dimensional heat transfer process is described by the equation: $\frac{\partial T(x, t)}{\partial t}=\frac{k}{c_{t} \rho_{t}} \frac{\partial^{2} T(x, t)}{\partial x^{2}}$,

where: $c_{t}$-specific heat, $\rho_{t}$-mass density, $k$-thermal conduction. This general heat transfer model does not include convection neither heat delivered by blood perfusion. Therefore temperature distribution and its change in time during and after excitation in biological tissue are described by the bio-heat transfer equation proposed by Pennes [12]:

$c_{t} \rho_{t} \frac{\partial T(x, y, z, t)}{\partial t}=k \nabla^{2} T(x, y, z, t)+Q_{b}+Q_{m}+Q_{z}$,

where: $k \nabla^{2} T(x, y, z, t)$-volumetric power density exchanged due to temperature gradient and thermal conduction, $Q_{b}$-volumetric power density exchanged due to blood perfusion, $Q_{m}$-volumetric power density caused by metabolism, $Q_{z}$-volumetric dissipated power density of an external heating source.

Unfortunately long execution time necessary for numerical solution of thermal problems which has to be acceptable in research is not practical in clinical conditions. Therefore taking into account expectation of medical staff instead of time consuming 3D and even 2D FEM modelling a simple approximation for each pixel by the exponential function is possible. Usually the two-exponential function is sufficient for practical applications, as accuracy of fitting measurement and model data is in this case almost perfect. For pulsed cooling, at the natural recovery phase the appropriate function for each pixel is expressed by:

$$
\begin{aligned}
T(x, y, t)= & T_{\min }(x, y)+\Delta T_{1}(x, y) \cdot\left(1-e^{-t / \tau_{1}(x, y)}\right) \\
& +\Delta T_{2}(x, y) \cdot\left(1-e^{-t / \tau_{2}(x, y)}\right),
\end{aligned}
$$

where symbols shown in Fig. 2a here are: $\mathrm{T}_{\min }=\mathrm{T}_{\mathrm{e}} ; \Delta \mathrm{T}_{1}+$ $\Delta \mathrm{T}_{2}=\left(\mathrm{T}_{0}-\mathrm{T}_{e}\right) ; \tau_{1}$ and $\tau_{2}$ respectively shorter and longer time constants; $\mathrm{T}_{0}$ - static temperature (before cooling).

Fortunately usually there are strong correlations between simple parametric model descriptors and theirs medical diag- 
nostic values. In the following discussion mainly such simple approach and presentation of parametric images of time constants as Figures of Merit for classification of treatment is applied. It has to be underlined that more complicated models in practice are not acceptable by clinicians.

For illustration the following paragraph shows two cases of new quantitative, objective methods - the skin burn diagnostics and evaluation of the post-cardiosurgery wound healing.

\section{Clinical Examples}

\subsection{Skin Burn Diagnostics}

Here we present results of several earlier research projects devoted to solve problems of IR-thermal imaging in skin burn diagnostics [13-17], but with data and images corrected after introduction of several procedures developed recently. Important aim of this discussion is to show typical for ADT IR-thermal imaging problems existing in biomedical diagnostics but not known in technical NDE applications. As the result functional static thermography and structural active dynamic thermography are compared and a new thermal diagnostic method allowing early objective determination of the burn region to be surgically treated is presented.

Proper evaluation of the surface and depth of a burn wound, especially in the case of a severe burn, enables an appropriate choice of conservative treatment or surgical intervention. The evaluation of the burn surface is not a difficult matter, but the assessment of burn depth remains a difficult problem and one that has not been fully resolved. Clinical assessment-visual observation-is currently the most frequently applied method in burn depth evaluation. Unfortunately the use of this method results in a high number of false diagnoses. Histopathology assessment of burn wound biopsies is the only reference approach owing to its high degree of accuracy, unfortunately the shortcomings_-invasiveness and long time of getting results restrict its usefulness in clinical practice.

The modern approach to burn treatment requires decisions as early as possible, but not later than at the second day after burn accident-indicative is the time of recovery-the wounds that will spontaneously heal within 3 weeks should be left for conservative treatment while the other require surgical intervention. Unfortunately till our research there did not existed any objective quantitative methods allowing foreseeing the result of treatment in 3 weeks of so called undetermined burns. One of the most promising method is laser Doppler imaging (LDI) [18] but none of methods is directly decisive in terms of treatment.

The value of any new diagnostic method should be carefully tested in objective fully controlled experiments. Often, as in our case [15-17], this is the crucial role of in vivo experiments on animals. The discussed projected work was accepted by the Local Ethics Committee for Animal Experiments of the Medical University of Gdansk. Results of in vivo animal experiments on eleven 6-week-old white Polish landrace domestic pigs, each weighing approximately $20 \mathrm{~kg}$ are presented. Analysis was made of 64 burn wounds inflicted according to the modification of Singer's procedure [19] and 22 reference areas of unburned skin. The analysis also made use of bacteriological methods. Additional histopathologic assessment data taken at symmetrically inflicted burns and reference fields allow to "calibrate" severity (depth) of burns, expressed as the relative thickness of the skin, a percentage of the dermis thickness at the measurement site (dtms), characterised by the following data for each of four types of burns: $1-11.92 \pm 2.98 \%$ dtms; $2-21.58 \pm 3.22 \%$ dtms; $3-47.80 \pm 18.16 \%$ dtms; $4-67.30 \pm 14.65 \%$ dtms. Further thermal analysis of chosen burns is shown in Fig. 7: a photographs of 4 burns at the second day after infliction; b 3 weeks later-fields $1 \& 2$ are healed; and parametric ADT images after cooling taken at the second day $\mathrm{c}-\mathrm{g}$. The reason for the choice of the pig as an experimental animal is the high degree of functional and structural similarity of pig skin to human skin [20].

The animal experiment results obtained were subjected to statistical analysis by means of the ANOVA variance analysis method and by comparing the average post hoc values with the Tukey's honestly significant difference (HSD) test [21]. The accuracy, sensitivity and specificity of the methods tested with reference to the characteristic sought, namely healing of the wound within 3 weeks of the burn, have been quantitatively calculated. We summarized the analysis by choosing the second day after burn as optimal for diagnostics and the second time constant as the most sensitive Figure of Merit.

When the clinical method of discriminating grades of depth of burn wounds was applied and a prognosis of healing result (and, in consequence, the mode of treatment selected) based on this, the calculated accuracy and sensitivity were low, at 62.5 and $44.2 \%$ respectively, but the specificity, at $100 \%$, was incommensurably high. These properties betray the limited usefulness of the method. When histopathologic assessment was employed, the total number of accurate prognoses (the measure of the accuracy of the method) rose to $89.1 \%$ and sensitivity to $97.7 \%$, while specificity decreased to $71.4 \%$. From these results it may be concluded that the usefulness of the histopathologic assessment of burn depth is considerably greater than that of the clinical method. The discussed problem is illustrated in Fig. 8.

By evaluating burn wound depth by means of ST and ADT modalities - it is claimed that ST improves on the power of clinical assessment to classify burns into groups, yet not to a fully satisfactory degree, because it does not discriminate the IIa from the IIb group. Instead, the values obtained for 
(a)

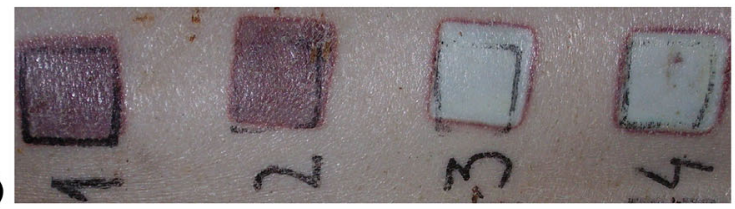

(b)

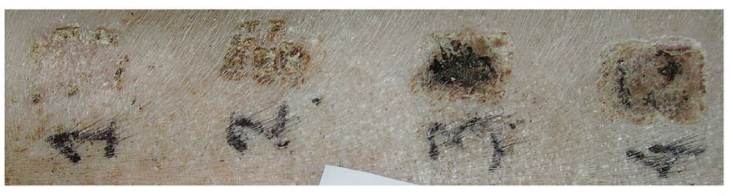

(c) 20

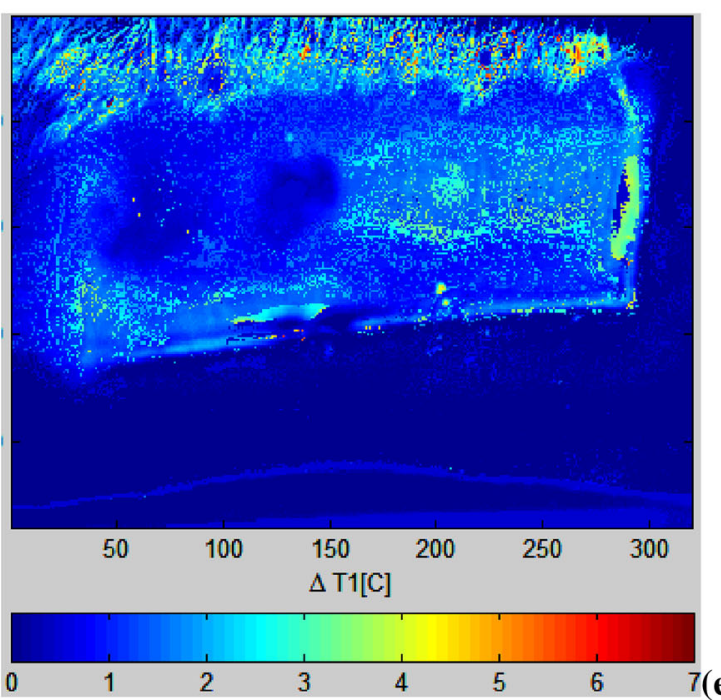

(d)

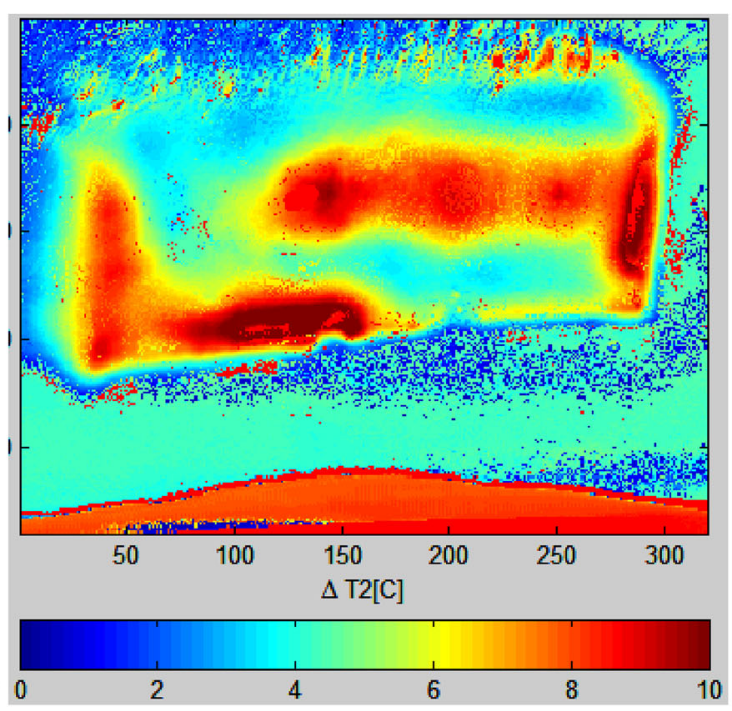

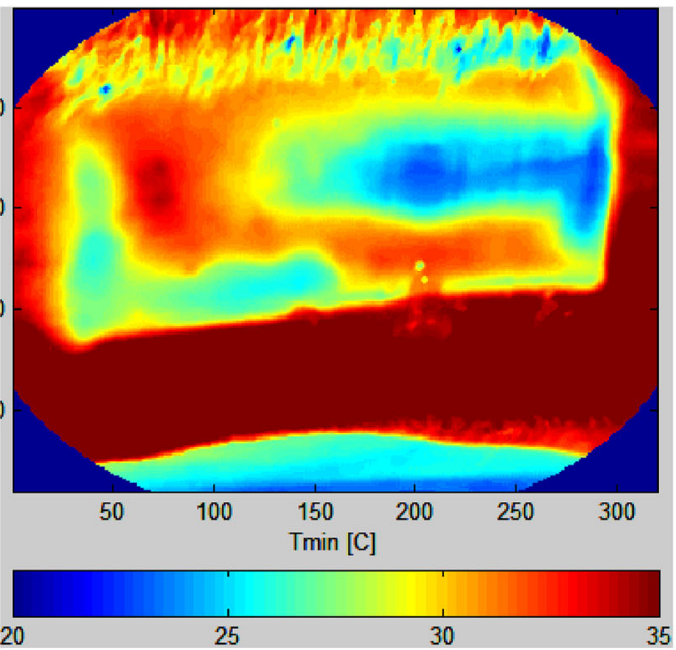
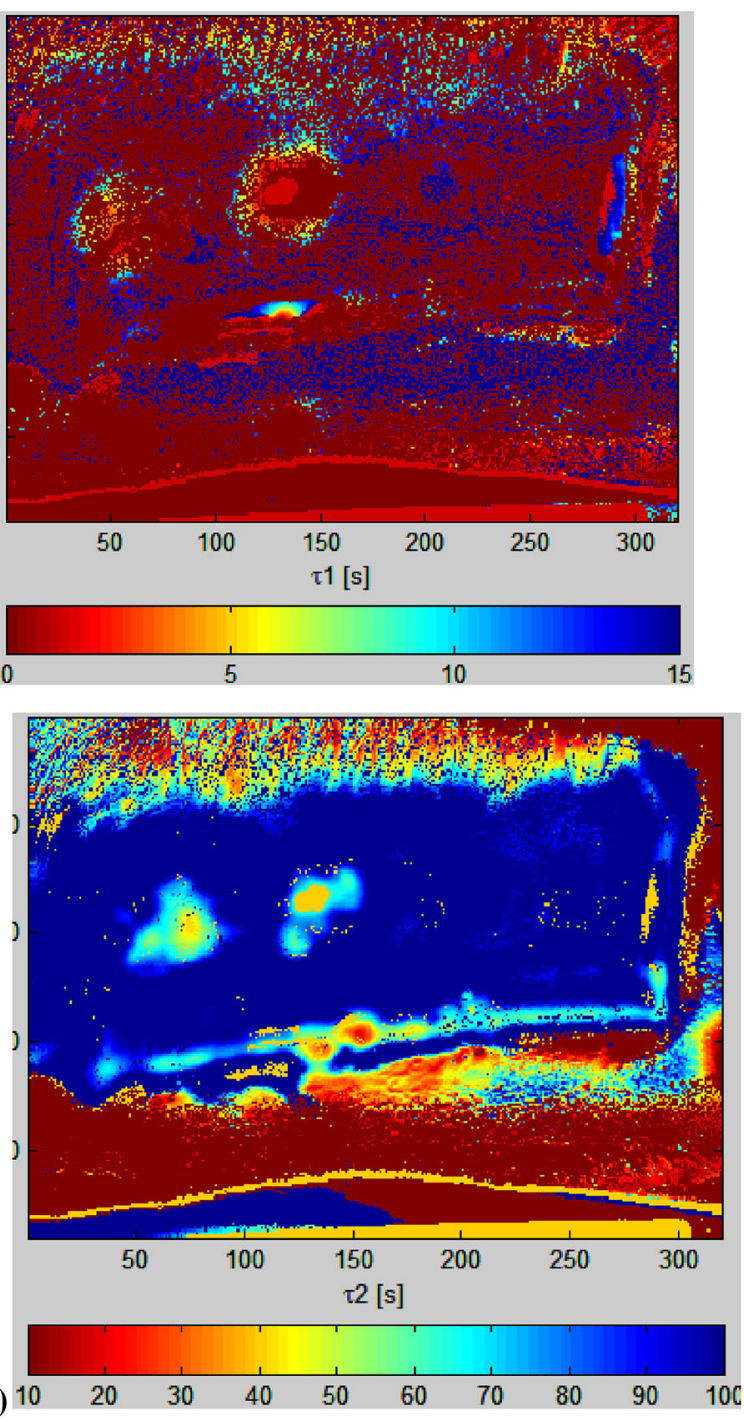

Fig. 7 In vivo experiment with pigs-four inflicted burns: a the RGB photo taken at the 2-nd day, b 21 days after burn infliction; and parametric images of the two-exponential ADT thermal model (the Eq. 3) for $\mathrm{CO}_{2}$ cooling $\mathbf{c} \mathrm{T}_{\min }=\mathrm{T}_{\mathrm{e}} ; \mathbf{d} \Delta \mathrm{T}_{1} ; \mathbf{e} \tau_{1} ; \mathbf{f} \Delta \mathrm{T}_{2} ;$ and $\mathbf{g} \tau_{2}$ (Color figure online) 
(a) ${ }^{-3}$

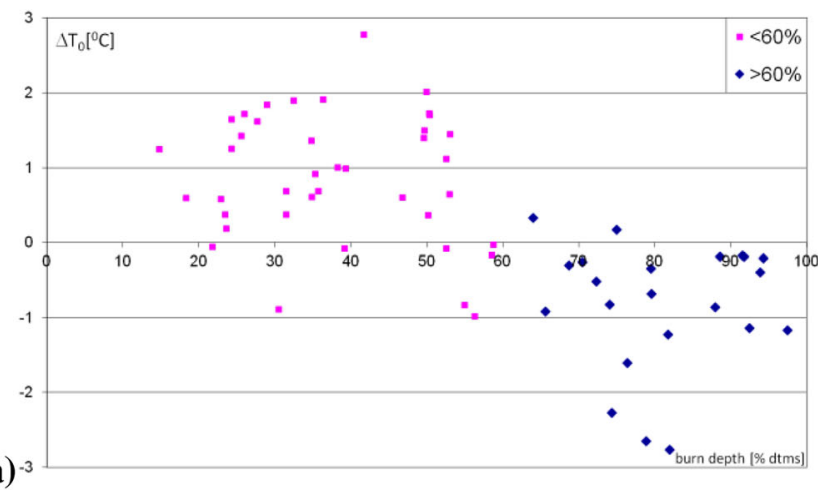

(b)

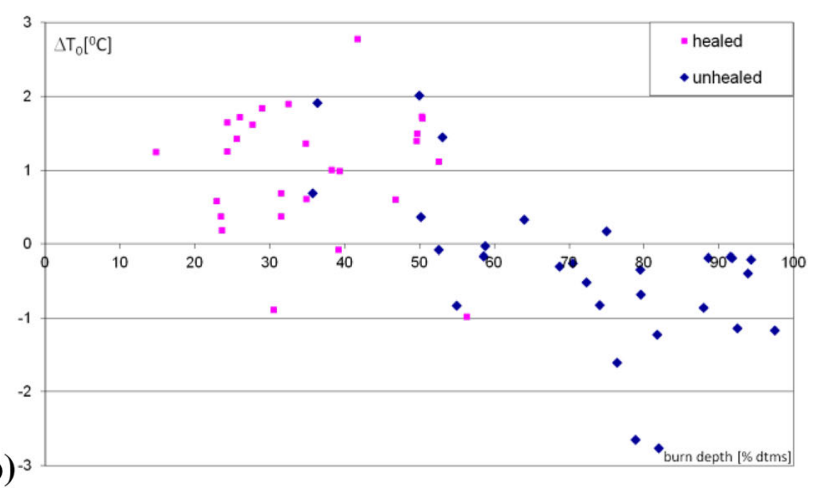

Fig. 8 Static temperature difference $\Delta \mathrm{T}_{0}$ vs burn depth: a according to burn depth criterion $60 \%$ dtms; $\mathbf{b}$ according the healing process after 3 weeks; all presented data are taken at the second day after inflicted burns
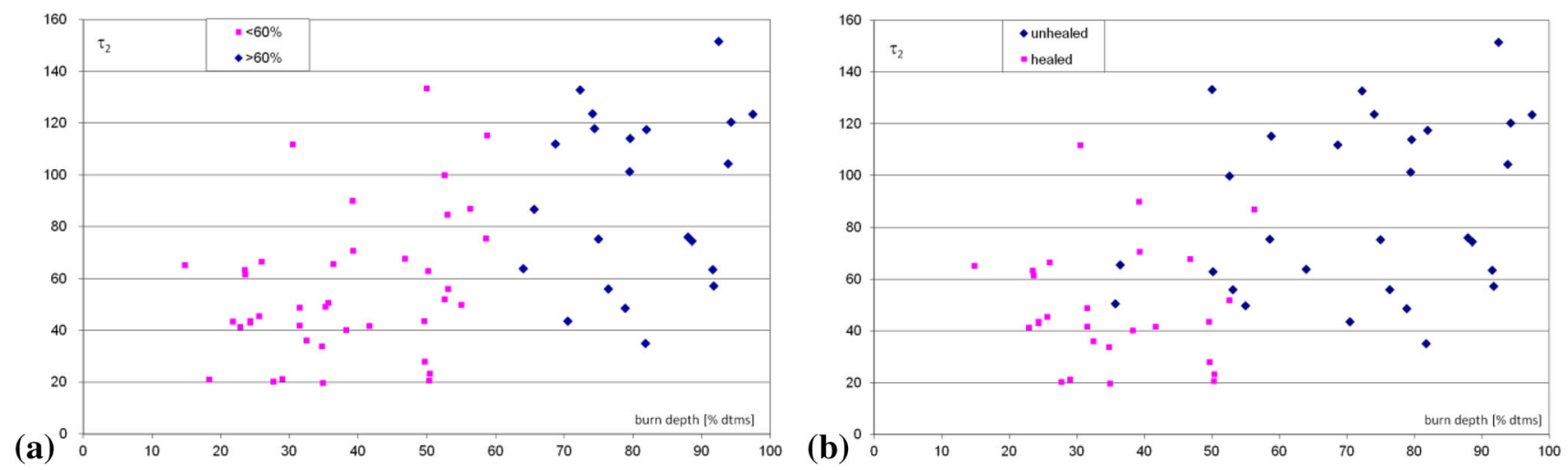

Fig. 9 Time constant $\tau_{2}[\mathrm{~s}]$ vs burn depth: a according to burn depth criterion $60 \% \mathrm{dtms}$; b according the healing process after 3 weeks; there is very hard to set the threshold value for $\tau_{2}$ parameter dividing the burns on two sets "unhealed" and "healed"; diagnostic interpretation of results remains difficult

the ST parameter, $\Delta T_{0}$ for both the groups involved when histopathologic assessment was used, differed at the level of statistical significance, $\mathrm{p}<0.001$. This demonstrates the high classificatory power of both methods (ST and histopathological evaluation). The best results were obtained by correlating the ST method with the expost classification into wounds that did heal within 3 weeks following infliction and those that did not heal spontaneously during this period. The threshold value of the $\Delta T_{0}$ parameter obtained, $\Delta T_{0}=0.3^{\circ} \mathrm{C}$, enabled a "prognostic" classification to be made of the burn wounds into those that would heal within 3 weeks of burning and those that would not heal spontaneously in this time and, in consequence, also enabled a proper choice to be made of a method of treatment. The mean values calculated for the synthetic ATD parameter $\tau$ did not differentiate the burn wounds classified into clinical groups at the level of statistical significance. In contrast, however, they did differentiate in this way the groups established according to the histopathological criterion (shallower or deeper than $60 \%$ of $\mathrm{dtms}$ ). This is an advantageous result because the histopathological assessment is recognised as the reference for the newer methods becoming established for the evaluation of burn wound depth. When the ADT method is employed, the results of classification based on the histopathological criterion (60\% of $d t m s$ ) are identical to these based on the clinical criterion "healing within 3 weeks".

It was very difficult to calculate the threshold value for $\tau_{2}$ parameter-see Fig. 9, but definition of the derivative normalized parameter formula (4)_-"normalized time constant":

$\tau_{2 n \_i}=\frac{\tau_{2 \_} i-\tau_{2 \_r e f}}{\tau_{2 \_} i+\tau_{2 \_r e f}}$,

where: $\tau_{2 \_} \mathrm{i}$ - the time constant for $\mathrm{i}$-th pixel in the parametric image, $\tau_{2}$ ref - the averaged time constant for unburned tissue chosen in a reference region, makes such discrimination of threshold value possible. The value of the normalized thermal time constant used in our work as the discriminator parameter is marked as $\tau_{2 \mathrm{n}} \mathrm{i}$, and shown in Fig. 10. The calculated threshold value of the normalized time constant is $\tau_{2 \mathrm{n} \text { _treshold }}=0.000113$ and the quality parameters of the method are as: accuracy-100 \%, sensitivity-100\% and specificity-100\%. In the interpretation of these unusually advantageous results necessary caution should be maintained because of the relatively small number of cases. 

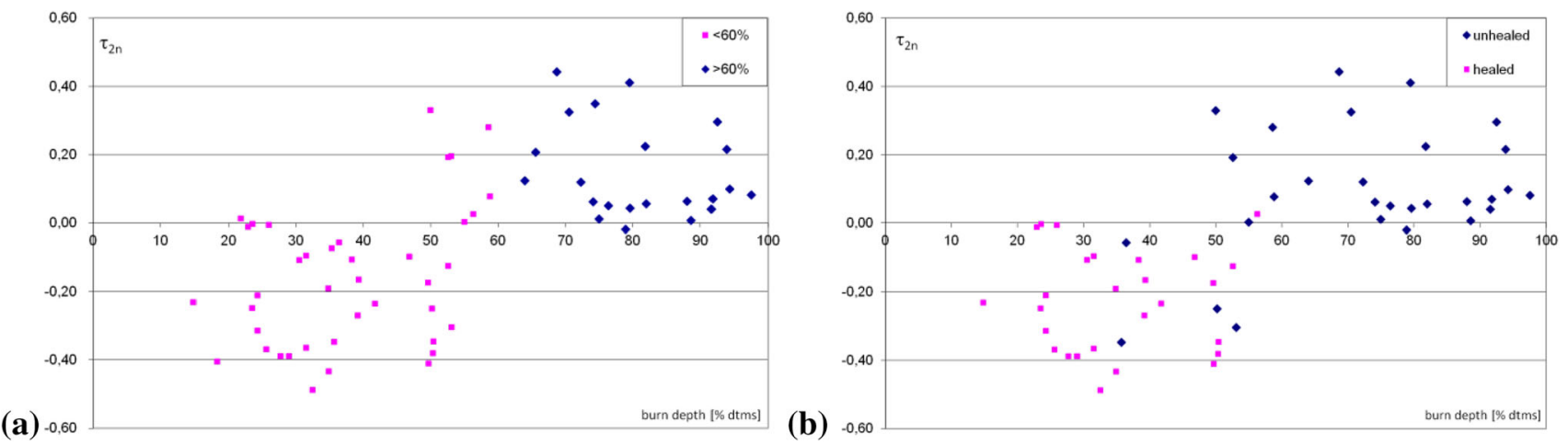

Fig. 10 Normalized time constant $\tau_{2 n}$ vs burn depth: a according to burn depth criterion $60 \%$ dtms; $\mathbf{b}$ according the healing process after 3 weeks; the best score for diagnostic decision

Table 1 Quality parameters for the sought feature of 3-week wound healing by the 4 methods evaluating the depth of all evaluated burn wounds [15]

\begin{tabular}{lllll}
\hline Quality parameters of the method & Clinical (\%) & Histopathologic (\%) & ST (\%) & ADT (\%) \\
\hline Accuracy & 60.90 & 100 & 91.30 & 100 \\
Sensitivity & 50.00 & 100 & 94.40 & 100 \\
Specificity & 100 & 100 & 80.00 & 100 \\
\hline
\end{tabular}

It should be underlined that the longer time constant $\tau_{2}$ and normalized derivative $\tau_{2 n}$ for applied excitation (cooling) correlate with properties of deeper tissue layer of the depth 1-2 mm. For burns that heal spontaneously within 3 weeks $\tau_{2 n}=-0.227 \pm 0.149$, (mean depth of burn = $35.24 \% \mathrm{dtms} \pm 11.18$ ), while for burns that did not heal $\tau_{2 \mathrm{n}}=$ $0.091 \pm 0.188$, (mean depth of burn $=72.12 \% \mathrm{dtms} \pm 17.67$ ). The threshold calculated by ANOVA analysis for classification to both groups equals $0.000113, \mathrm{p}<0.050$, Fig. $10 \mathrm{~b}$.

The comparison of tested methods is given in Table 1.

In presenting the possibility of applying ADT as a quantitative method of burn depth evaluation, suitable thermal models of unburned skin and of burn wounds of different depths have been proposed. The first is the simple analytical model, which can be used in clinical practice. The second, a numerical one, is more complex and serves for the analysis and explanation of the correlation between the accepted synthetic parameter and the features and processes occurring in unburned skin as well as in burn wounds. It has been shown that the simulation of the measuring process employed in the ADT method, when performed on the numerical models elaborated of unburned and burned skin, enables the values of parameters to be obtained during the thermal recovery phase which are analogous to the measurement results obtained by means of this method.

The results of an investigation into human burn wounds by means of thermographic methods involving several patients treated in the Department of Plastic Surgery of the Medical University of Gdansk-GUMed - have been also presented (by permission of the Independent Bioethics Committee on Scientific Investigation of GUMed). Here the case of a 19 year old cook burned by boiling oil is shown as an example.

The histopathology of the biopsy specimen taken in the center of the undetermined burn area was $50.4 \% \mathrm{dtms}$. This result means that this part of the wound should heal within 3 weeks. Meaning of the indicated marks in Fig. 11:

1-superficial burn, which will heal within 3 weeks,

?-undetermined burn wound,

$\mathrm{Ub}$ - unburned area.

The undetermined in visual inspection part of the wound was accurately diagnosed by means of the ADT method. The whole abdomen area was healed in 3 weeks. Figure 12 shows the ADT parametric images. All area are classified as to be healed spontaneously within 3 weeks.

One may interpret the images after training in analysis of ADT images. In Fig. 12b the parametric image of the normalized time constant $\tau_{2 n}$ (Eq. 4) shows relatively uniform distribution of this Figure of Merit (close to zero value or above zero) what should be interpreted as predicted natural healing of the full burn wound. This simple quantitative decision criterion is fully accepted by medical staff.

\subsection{Evaluation of Cardiosurgery and Post-cardiosurgery Wound Healing}

This application example is relating results of the recent research grant $[22,23]$, project financed by the Polish National Science Centre devoted to evaluation of cardiosurgery wound healing based on analysis of thermal tissue 
(a)
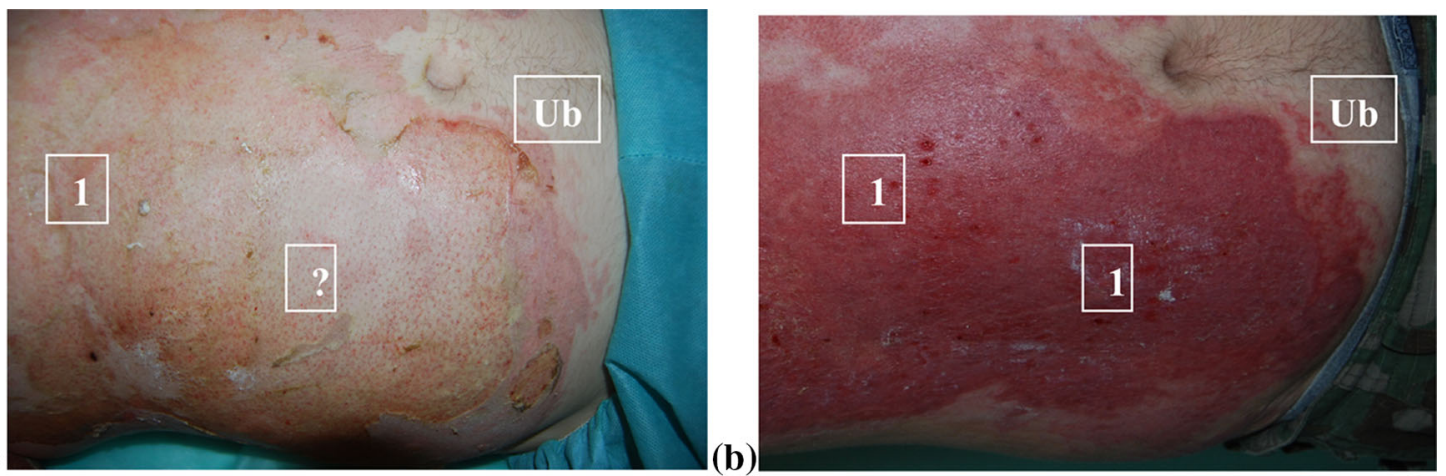

Fig. 11 Clinical application - a burn wound to the abdomen in 19 year old man-RGB photos taken a at the 2-nd day and b 21 days following burn accident

properties using QIRT approach. As the final results of this project the development of a new objective and quantitative method of post cardiosurgery wound healing diagnostics is presented. The problem of healing post-cardiosurgery wounds from medical perspective is described in details in [24]. We assumed that thermal processes should be effective in subtle description of temporal changes of tissue thermal properties after surgical interventions. We applied both, ST the classical thermal image capture as well as ADT active dynamic thermography method, for analysis of thermal processes existed in the vicinity of the postoperative wound.

We performed in the project examination on ca 400 patients after cardiosurgery intervention on the open heart participated in clinical experiments using IR thermal cameras as diagnostic instrumentation, searching mainly for complications in healing. The procedure comprises all possible diagnostic information including ST and ADT data at the day before operation (day 1), and then ST and ADT at the day after operation (day 3) and 3 days later (day 6). For statistic analysis based on quantitative data we have chosen 20 cases belonging to the group without complications, to compare different descriptors as potential Figures of Merit for classification of patients. This group is regarded as the reference data. The results of this group are gathered in Table 2 showing mean values of all chosen descriptors and its' standard deviation (sd). Unfortunately information of the reference group of patients is limited as those patients are discharged from a hospital as soon as possible, usually at the 5-th day after operation.

As it was discussed in the paragraph 3 in the project several packages of software prepared for data analysis are applied. The first one is devoted to geometry corrections, matching of images taken in consecutive days of treatment and determination of the same ROI in all images. The next one is for denoising/filtration of images. Also matching of different modality images is important in qualitative evaluation of the content. Usually thermal images are of a low geometry resolution so matching with visual image allows for more precise interpretation of diagnostic content as it is shown in Fig. 13. As an example a result of matching of a color photograph and a thermogram with additional determination of the ROI and parametric image of the descriptor in ROI is shown.

All calculations of parametric images according to equation (3) are performed only for matched ROI region on sequences of thermograms, so results of visualization show this same region on the patient chest surface.

We choose as quantitative parameters for evaluation of the wound healing process the differences between day 6 and day 3 :

$$
\begin{aligned}
& \Delta T_{\min }=T_{\min } \text { (6day) }-T_{\min }(3 \text { day }), \\
& \Delta \tau_{2}=\tau_{2} \text { (6day) }-\tau_{2}(3 \text { day }) .
\end{aligned}
$$

As an example of diagnostic procedure images of a chosen patient with complications in healing is presented in Fig. 14, according to the model (3). To show the score of classification only the pixels closest to the wound are taken into accountthe region $\pm 10 \times 210$ pixels, averaged values in segments $10 \times 5$.

The images are in columns representing data of the days 1, 3 and 6. The rows represent: a a photograph, $\mathrm{b} \mathrm{T}_{\text {min }}, \mathrm{c}$ segmented $\mathrm{T}_{\min }, \mathrm{d} \tau_{2}$, e segmented $\tau_{2}$, f the differential images defined by (5).The result of diagnostics is presented as the score, at the end of the Fig. 14.

According to Table 2 the value of the $\tau_{2}$ parameter of the two exponential model is chosen as the decisive Figure of Merit. For the $\Delta \tau_{2}$ parameter the three stage classifier and classification thresholds are set as:

$$
\begin{array}{lll}
-1 & \text { for } & \Delta \tau_{2}<-10 \\
0 & \text { for } & -10<\Delta \tau_{2}<1 \\
1 & \text { for } & \Delta \tau_{2}>10
\end{array}
$$

represented on the chart (Fig. 14) as the score:

black color (-1)—means "good (well) healing process"; 

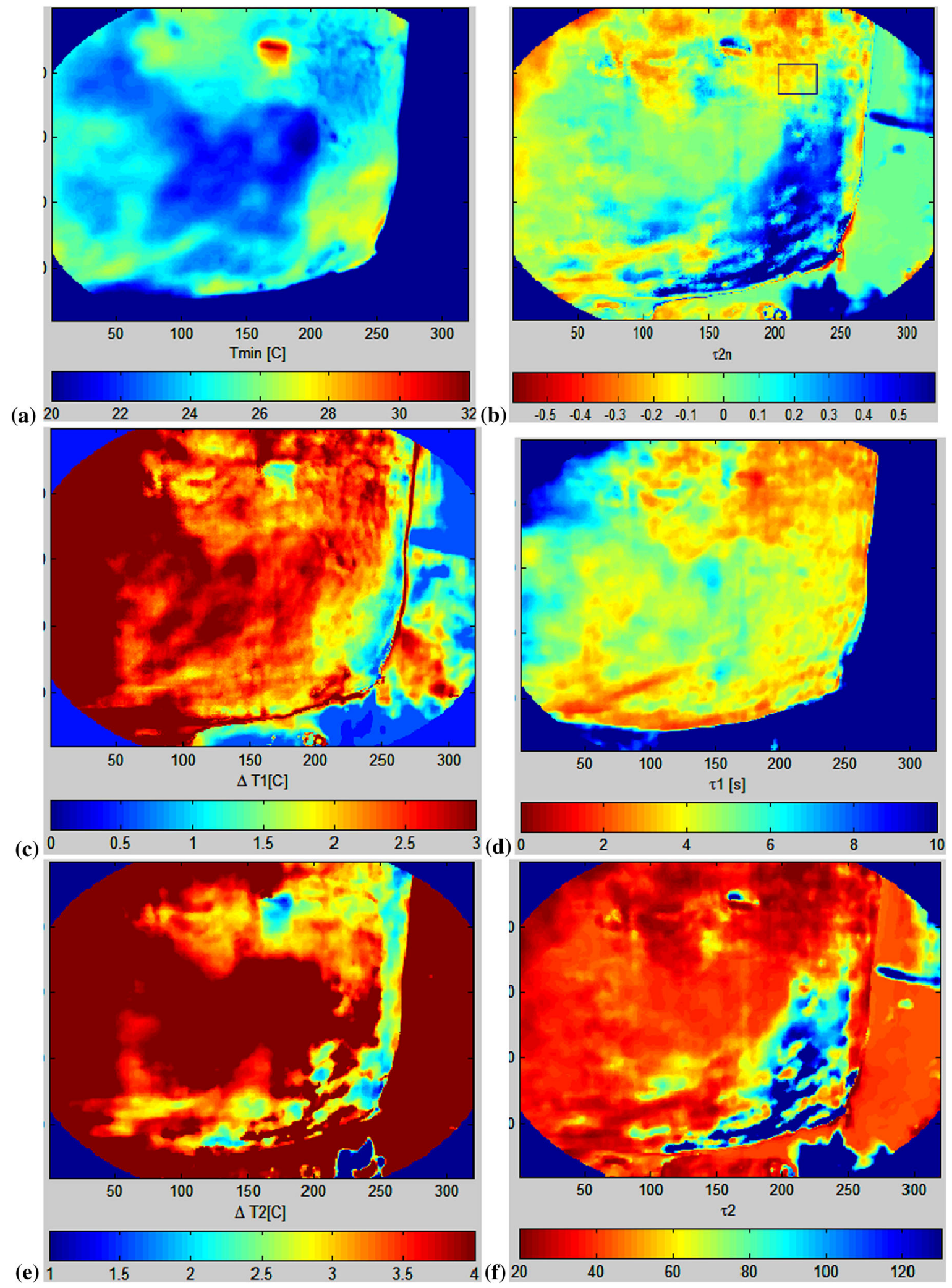

Fig. 12 Clinical case from Fig. 11-ADT parametric images of the two-exponential thermal model for $\mathrm{CO}_{2}$ cooling: a temperature at the moment of cooling termination; $\mathbf{b}$ derivative normalized time constant

for the reference region indicated in the rectangle-Eq. 4; $\mathbf{c}$ and $\mathbf{e}$ temperature components from Eq. 3; $\mathbf{d}$ and $\mathbf{f}$ time constants (Color figure online) 
Table 2 Mean values of descriptors at ROI for the reference group of 20 patients

\begin{tabular}{|c|c|c|c|c|c|c|c|}
\hline \multirow[t]{2}{*}{ Descriptor } & \multicolumn{2}{|c|}{ Day 1 reference data } & \multicolumn{2}{|l|}{ Day 3} & \multicolumn{2}{|l|}{ Day 6} & \multirow{2}{*}{$\frac{\mathrm{d} 6-\mathrm{d} 3}{\text { Value }}$} \\
\hline & Value & $\mathrm{Sd}$ & Value & $\mathrm{Sd}$ & Value & $\mathrm{Sd}$ & \\
\hline $\mathrm{T}_{\min }\left[{ }^{\circ} \mathrm{C}\right]$ & 33.42 & 1.36 & 34.34 & 1.10 & 33.75 & 1.03 & -0.59 \\
\hline$\tau_{1}[\mathrm{~s}]$ & 3.40 & 1.15 & 3.36 & 0.97 & 3.76 & 1.23 & 0.40 \\
\hline$\tau_{2}[\mathrm{~s}]$ & 35.60 & 8.90 & 32.07 & 8.03 & 38.49 & 9.88 & 6.41 \\
\hline
\end{tabular}

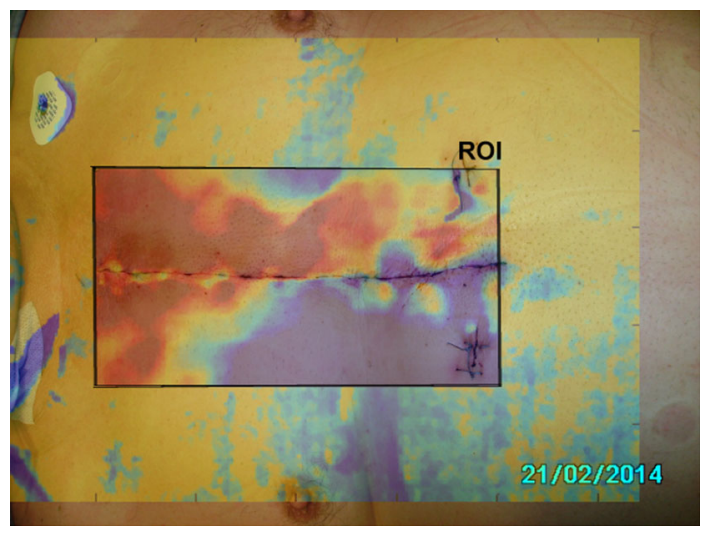

Fig. 13 Procedure of choosing ROI-first, RGB and thermal images are corrected and matched; second, the region of interest is defined at the day 3 , defined in pixels- \pm 40 pixels symmetrical along the wound of 210 pixels; third, the selected ROI should be inserted to all diagnostically significant images taken at all days of diagnostics

gray color (0)—means "hard to say";

white color (1) - means "bad (poor) with complication healing process".

Figure 15 shows typical diagnostic result. The score is presented on the matched photo and parametric image of $\tau_{2}$. Then the diagnostic decision is-keeping the patient at the hospital for further treatment.

In most of clinical cases the day 6, 4 days after operation, is decisive in terms of keeping the patient for further treatment in the hospital or discharging for rehabilitation at home. The main problem to get statistically significant research result is a small number of complicated healing cases.

The discussed project in fact is summarizing our earlier extensive research on quantitative analysis of the quality of cardiosurgery operations $[25,26]$. One of the important questions we investigated was minimization of the risk of cardiosurgery procedures. Due to high risk of cardiosurgery interventions analysis of some procedures was possible only based on animal experiments. In the following Fig. 16 an interesting example of measurements during cardiosurgery open chest interventions [26] is illustrated for in-vivo experiments on domestic pig. For detailed discussion please refer to the original publications where some ADT experiments on pigs as well as clinical cases are also presented.

\section{Discussion and Summary}

In this paper we presented new in medical applications procedures and diagnostic methods based on active IR-thermal imaging and external tissue excitation using mainly cooling cryotherapy instrumentation. This approach is discussed in perspective of well known classical thermography. As the effect multimodality approach is proposed as an important improvement of thermal diagnostics quality.

As a practical solution we propose the use of very simple two-exponent model and determination of parametric images as Figures of Merit. Our medical experiments show, that the second time constant is more sensitive while cooling than other possible parametric descriptors, also the cooling gives more informative diagnostic data comparing to heating. The problem of thermal excitation parameters is also very important. Magnitude and duration of the cooling or heating phase is crucial, as the interacting energy must be limited to avoid any danger interaction with the living organ; also time of excitation must be minimised to avoid bio-feedback interactions, difficult for proper interpretation of heat flows. On the other hand duration of the excitation should be properly long to give clear discrimination of deep structures including burns or post-operative wounds.

One of the most important for understanding thermal flows in living tissue is the question of non-uniform temperature distribution. Usually internal temperature is decreasing in closer to superficial layers of tissue. This difference might be even several degrees per millimetre. In effect cooling is much more practical in ADT experiments, especially in the case to stop cooling at the room temperature, we follow.

In bio-medical experiments the first requirement is always to show quantitative results done in fully controlled conditions. Therefore results of in vivo animal experiments (using pigs that have not only skin but also the heart and the vascular system almost identical to human) are of high value in early establishment of new measurement methods, we are seeking for. Always the main question is-how to understand the results of thermal experiments? This question is not trivial, as interpretation of heat flows strongly depends on boundary conditions. In fact we have shown [15], that due to temperature gradient existing in a living mammals the skin temperature is usually lower than internal tissue. Increasing 


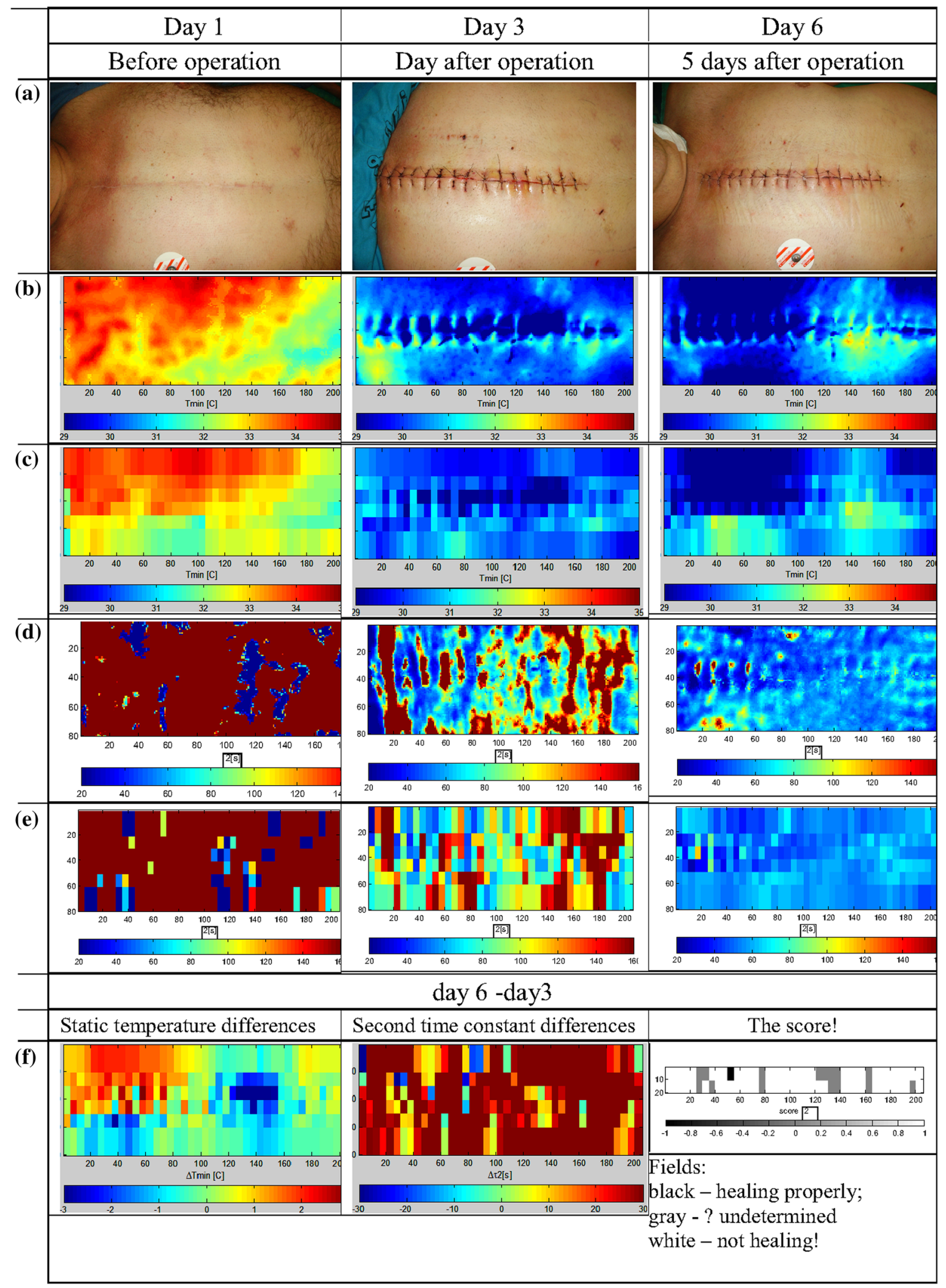

Fig. 14 Most important images for analysis of cardiosurgery wound healing, here the patient with complications, negative opinion of healing at the 6-th day-decision-further treatment in the hospital (Color figure online) 


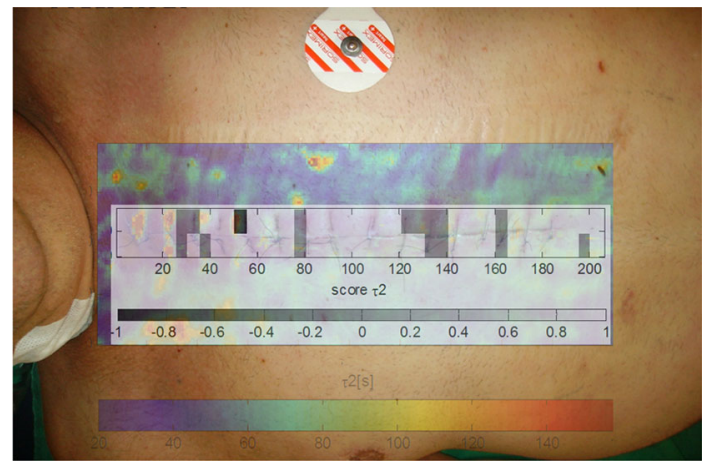

Fig. 15 Visualization of final results: fusion of photo and parametric images: $\tau_{2}$ and score $\tau_{2}$ for analysis of cardiosurgery wound healing (Color figure online)

this temperature by heating makes the internal temperature gradient lower. In this case usually heat exchange is mainly to the environment, and is not very strongly dependent of the internal skin structure, what is our concern in burn diagnostics but also in other applications. Cooling gives much more reliable data of the tested skin as temperature gradient is increased and flow is mainly inside a tested structure.
Analysis of both-the ST as well as the ADT measurement results leads to the conclusion that special care must be taken to secure proper measurement conditions. Important is also process of experiment simulation necessary in reconstruction of a tested structure, e.g. using Thermal Tomography algorithms, as it was earlier underlined [27].

The clinical results of the work are very promising as the developed of versatile, objective instrumentation allows for qualitative and quantitative evaluation of many superficial symptoms of illness, as burns-depth and region of surgical interventions; skin transplants; breast reconstruction surgery control, evaluation of post surgery wound healing and other. Promising applications concern evaluation of surgical procedures, including cardiosurgery, mammography and many other.

In summary - ST and ADT are different modalities implementing each other. The combined multimodality data are of much higher specificity, accuracy and sensitivity in many medical investigations. Further matching of other medical imaging methods is a great promise for modern medicine. The decreasing costs of IR-thermal imaging makes very optimistic prognosis of multimodality approaches in clinical applications.

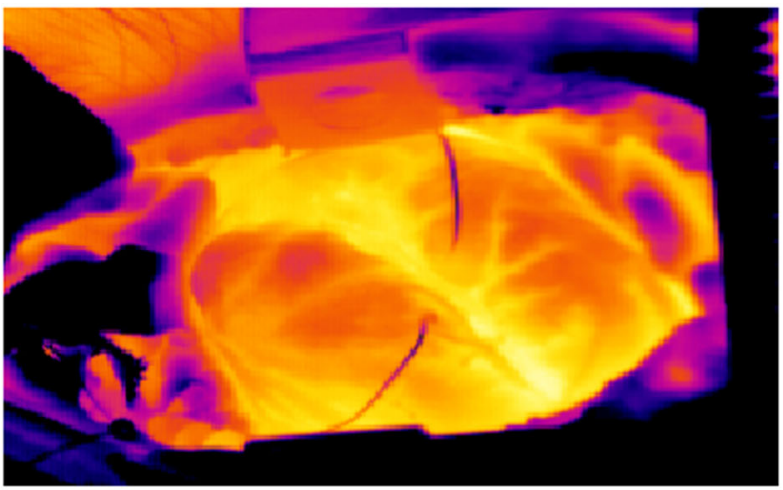

$0 \mathrm{~s}$

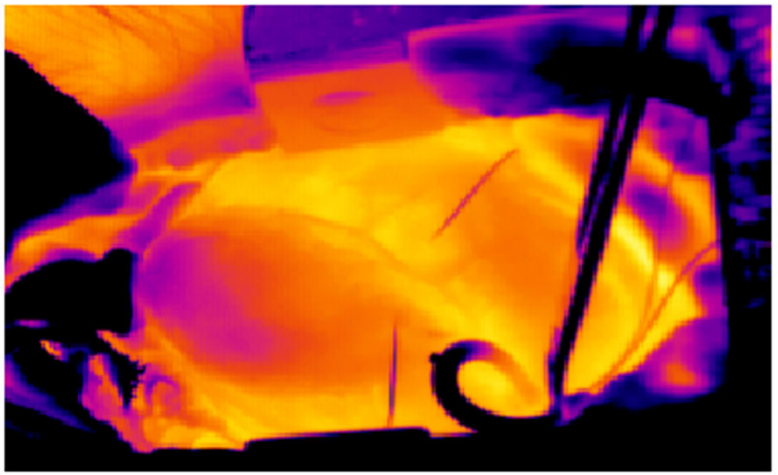

$30 \mathrm{~s}$

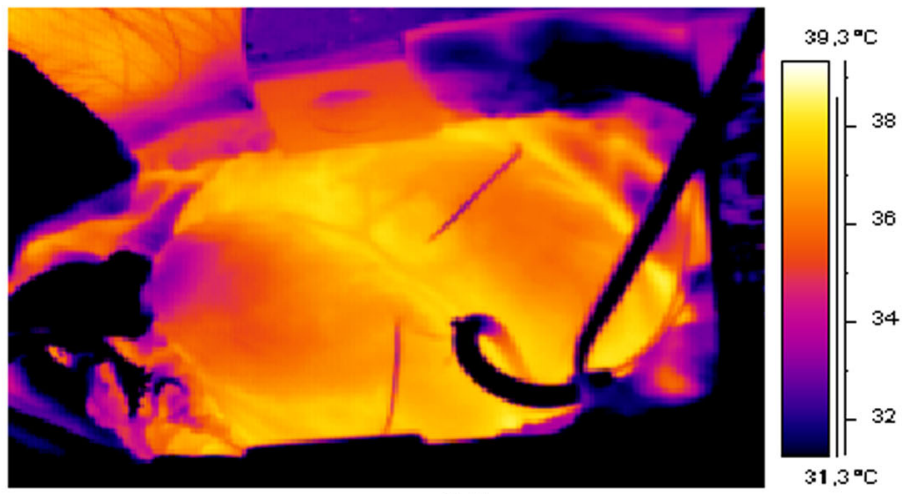

$10 \mathrm{~s}$
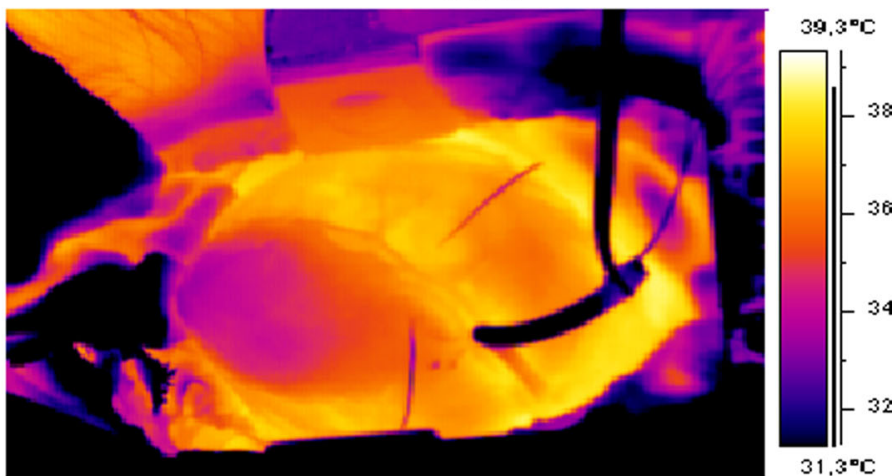

$60 \mathrm{~s}$

Fig. 16 The static thermograms of the pig heart taken at the indicated times after clamping LAD (left descending artery) what is equivalent to induced heart stroke; thermal camera is synchronised with the heart rate-ECG monitor; temperature at the affected region is slowly decreasing [26] (Color figure online) 
Acknowledgments Authors acknowledge participation in this work of all co-workers from the Department of Biomedical Engineering Gdansk University of Technology, from the Department of Plastic Surgery, the Department of Cardiac Surgery and other departments from Gdansk University of Medicine and from the Department of Animal Physiology Gdansk University, where animal experiments have been performed. This work was financed by several research grants, recently by the Polish National Science Centre NCN UMO2011/03/B/ST7/03423 Development of new IR-imaging descriptors for objective evaluation of post-operative wound healing and partly from GUT Faculty of Electronics, Telecommunications and Informatics statue funds.

Open Access This article is distributed under the terms of the Creative Commons Attribution 4.0 International License (http://creativecomm ons.org/licenses/by/4.0/), which permits unrestricted use, distribution, and reproduction in any medium, provided you give appropriate credit to the original author(s) and the source, provide a link to the Creative Commons license, and indicate if changes were made.

\section{References}

1. Maldague, X.P.V.: Theory and Practice of Infrared Technology for Non-destructive Testing. Wiley, New York (2001)

2. Vavilov, V.: Thermal NDT: historical milestones, state-of-the-art and trends. JQIRT 11(1), 68-83 (2014)

3. Diakides M., Bronzino J., Peterson D., (ed.): Medical Infrared Imaging-Principles and Practices, CRC Press (2013)

4. Ring F., Jones F.: Historical Development of Thermometry and Thermal Imaging, p. 2.1-2.6, ibid

5. Amalu W.C., Hobbins W.B., Head J.F., Elliot R.L.: Infrared Imaging of the Breast: A review, p. 10-1-10-22, ibid

6. Barnes, R.B.: Thermography and its clinical applications. Ann. N. Y. Acad. Sci. 121, 34-48 (1964)

7. Gershen-Cohen, J., Haberman, J., Bruesche, E.E.: Medical thermography: a summary of current status. Radiol. Clini. North Am. 3, 403-431 (1965)

8. Keyserlingk, J.R., Algren, P.D., Yu, E., Belliveau, N., Yassa, M.: Functional infrared imaging of the breast: historical perspectives, current applications, and future considerations. In: Diakides, M., Bronzino, J., Peterson, J. (eds.) Medical Infrared ImagingPrinciples and Practices, pp. 11-1-11-28. CRC Press, Boca Raton (2013)

9. Kaczmarek, M., Nowakowski, A., Suchowirski, M., Siebert, J., Stojek, W.: Active dynamic thermography in cardiosurgery. QIRT 4, 107-123 (2006)

10. Beier, T., Neely, S.: Feature-based image metamorphosis. Comput. Grap. SIGGRAPH 26(2), 35-42 (1992)

11. Moderhak, M.: FFT spectra based matching algorithm for active dynamic thermography. QIRT J. 8(2), 239-242 (2011)

12. Pennes, H.H.: Analysis of tissue and arterial blood temperatures in resting human forearm. J. Appl. Physiol. 1, 93-122 (1947)

13. Nowakowski, A., Kaczmarek, M., Rumiński, J., Hryciuk, M., Renkielska, A., Grudziński, J., Siebert, J., Jagielak, D., Rogowski, J., Roszak, K., Stojek, W.: Medical applications of model based dynamic thermography. Proceedings of Thermosense XXIII Conference, SPIE, vol. 4360, pp. 492-503 (2001)
14. Renkielska, A., Nowakowski, A., Kaczmarek, M., Dobke, M.K., Grudzinski, J., Karmolinski, A., Stojek, W.: Static thermography revisited - an adjunct method for determining the depth of the burn injury. Burns 31, 768-775 (2005)

15. Renkielska, A., Nowakowski, A., Kaczmarek, M., Rumiński, J.: Burn depths evaluation based on active dynamic IR thermal imaging - a preliminary study. Burns 32, 867-875 (2006)

16. Rumiński, J., Kaczmarek, M., Renkielska, A., Nowakowski, A.: Thermal parametric imaging in the evaluation of skin burn depth. IEEE Trans. Biomed. Eng. 54(2), 303-312 (2007)

17. Renkielska, A., Kaczmarek, M., Nowakowski, A., Grudziński, J., Czapiewski, P., Krajewski, A., Grobelny, I.: Active dynamic infrared thermal imaging in burn depth evaluation. J. Burn Care Res. 35(5), 294-303 (2014). doi:10.1097/BCR. 0000000000000059

18. Kloppenberga, F.W.H., Beerthuizena, G.I.J.M., Ten Duis, H.J.: Perfusion of burn wounds assessed by Laser Doppler Imaging is related to burn depth and healing time. Burns 27(4), 359-363 (2001). doi:10.1016/S0305-4179(00)00138-8

19. Singer, A.J., Berruti, L., Thode, H.C., McClain, S.A.: Standardized burn model using a multiparametric histologic analysis of burn depth. Acad. Emerg. Med. 7, 1-6 (2000)

20. Sullivan, T.P., Eaglstein, W.H., Davis, S.C., Mertz, P.: The pig as a model for human wound healing. Wound Repair Regen. 9(2), 66-76 (2001)

21. Abdi, H., Williams, L.J.: Tukey's honestly significant difference (HSD) test. In: Salkind, Neil (ed.) Encyclopedia of Research Design. Sage, Thousand Oaks (2010)

22. Nowakowski, A., Siondalski, P., Moderhak, M., Kaczmarek, M.: Problems of Cardiosurgery Wound Healing Evaluation, Proceedings of QIRT, pp. 1-9, Bordeaux (2014)

23. Nowakowski, A., Siondalski, P., Moderhak, M., Kaczmarek, M.: A new diagnostic method for evaluation of cardiosurgery wound healing, JQIRT, pp. 1-16 (2015). doi:10.1080/17686733.2015. 1077543

24. Siondalski P., Algorytm leczenia powikłań gojenia się ran pooperacyjnych i ropnego zapalenia śródpiersia po operacjach kardiochirurgicznych [Algorithm dealing with the treatment of healing wound complications and mediastinitis after cardiac surgeries]. Annales Academiae Medicae Gedanensis. 39(2), 303-413 (2009)

25. Kaczmarek, M., Nowakowski, A., Siebert, J., Stojek, W., Suchowirski, M.: Active dynamic thermography in cardiosurgery. Quant. InfraRed Thermogr. J. 4(2007), 107-123 (2007)

26. Nowakowski, A., Kaczmarek, M., Rogowski, J.: The role of thermal monitoring in cardiosurgery interventions. In: Diakides, M., Bronzino, J.D., Peterson, D.R. (eds.) Medical Infrared ImagingPrinciples and Practices, pp. 17-1-17-24. CRC Press, Boca Raton (2013)

27. Nowakowski, A.: Quantitative active dynamic thermal IR-imaging and thermal tomography. In: Diakides, M., Bronzino, J.D., Peterson, D.R. (eds.) Medical Infrared Imaging-Principles and Practices, pp. 7-1-7-30. CRC Press, Boca Raton (2013) 